'Programa de Salud Basada en la Evidencia, Escuela de Medicina, Pontificia Universidad Católica de Chile.

${ }^{2}$ Departamento de Medicina

Familiar, Escuela de Medicina, Pontificia Universidad Católica de Chile. Santiago de Chile.

Correspondencia a: Dr. Tomás Pantoja. Departamento de Medicina Familiar. Centro Médico San Joaquín. Avda. Vicuña Mackenna 4686, Macul, Santiago. Teléfonos: 3548535 - 3548688 tpantoja@med.puc.cl

\section{Guías de práctica clínica: una introducción a su elaboración e implementación}

\author{
TOMÁS PANTOJA ${ }^{1,2}$, MAURICIO SOTO ${ }^{1,2}$
}

\section{Clinical practice guidelines development and implementation: an introduction}

\begin{abstract}
Clinical Practice Guidelines (CPG), defined as "statements that include recommendations intended to optimize patient care that are informed by a systematic review of evidence and an assessment of the benefits and harms of alternative care options", are tools currently present in every level of our health system. This article introduces guidelines development and implementation processes and it reviews the Chilean experience. The main stages in a CPG development are question formulation, search and analysis of the existing evidence related to those questions, and making judgments about that evidence in order to formulate recommendations for clinical practice. At the national level, guidelines development processes are conducted by the Ministry of Health, and even when recent evaluations show some good results, there are a number of aspects - such as applicability - that should be improved. On the other hand, CPG should be implemented using effective strategies in order to obtain changes in clinical practice and patients' outcomes. The existing evidence about the effects of the different implementation strategies shows modest and highly variable results. At the national level, there is a dearth of research about the design and evaluation of implementation strategies, and most of it has been focused in the evaluation of adherence to specific recommendations.
\end{abstract}

(Rev Med Chile 2014; 142: 98-104)

Key words: Practice guidelines as topic; Review; Standards.
E n las últimas décadas la calidad de la atención en salud ha sido un tema permanente de debate en las políticas de salud de muchos países, y numerosos gobiernos han asumido la promesa de incorporar estrategias que mejoren dicha calidad ${ }^{1-3}$. En este contexto, el denominado "movimiento" de la Medicina Basada en Evidencia (MBE) ha jugado un importante rol en promover la toma de decisiones basadas en la mejor evidencia disponible y, por ende, contribuir a la mejoría de la calidad de la atención sanitaria ${ }^{4-6}$.

Sin embargo, la práctica de un cuidado de salud basado en la evidencia, con sus "clásicas etapas" (formulación de la pregunta, búsqueda de la infor- mación, análisis crítico, aplicación), parece no ser la alternativa preferida por muchos profesionales de la salud debido, en gran parte, a limitaciones en el tiempo para practicarla ${ }^{7-9}$. Por esta razón es que muchos profesionales de la salud, en especial en la atención primaria, prefieren utilizar resúmenes basados en evidencia generados por otros y Guías de Práctica Clínica (GPC) para la aplicación de los principios de la $\mathrm{MBE}^{7,10,11}$.

Las GPC son, de acuerdo a la definición del Instituto de Medicina de los Estados Unidos de Norteamérica, "enunciados elaborados sistemáticamente para asistir las decisiones de los profesionales sanitarios y sus pacientes acerca 
del cuidado de salud apropiado en circunstancias clínicas específicas"12. En una reciente publicación, esta misma organización precisa que las GPC son "enunciados que incluyen recomendaciones dirigidas a optimizar el cuidado de los pacientes y que están informadas por una revisión sistemática de la evidencia y una evaluación de los beneficios y daños de opciones de manejo alternativas"13.

Aunque en estricto rigor en la literatura académica relacionada con las GPC es posible identificar una serie de procesos que han sido descritos como elaboración, difusión, diseminación e implementación, habitualmente los tres últimos son incluidos en el concepto más amplio de implementación, debido a las diferentes interpretaciones de los conceptos de difusión y diseminación ${ }^{14,15}$. A pesar de dichas distinciones, los procesos de elaboración e implementación se encuentran íntimamente ligados, tanto desde el punto de vista práctico como teórico. La elaboración de guías sin considerar las estrategias a través de las cuales serán implementadas no producirá los efectos deseados en los profesionales sanitarios ni en los resultados de salud en los pacientes, así como tampoco lo hará la implementación de guías elaboradas a través de procesos poco rigurosos.

El presente artículo aborda de manera introductoria los procesos de elaboración e implementación de GPC, junto con revisar la experiencia chilena en cada uno de ellos.

\section{Elaboración de GPC}

Las etapas fundamentales en la elaboración de una GPC son la formulación de preguntas clínicas específicas, la búsqueda y análisis de la evidencia científica existente respecto a las preguntas específicas que la GPC aborda, y la realización de juicios respecto a dicha evidencia, los que se traducen en recomendaciones para la práctica clínica (Figura 1). El proceso también puede ser desagregado en un número mayor de etapas: i) identificación del tema de la GPC; ii) definición del equipo que elaborará la GPC, iii) identificación de las diferentes partes interesadas involucradas en la elaboración e implementación (audiencia objetivo y probables usuarios, entre otros); iv) formulación de las preguntas clínicas claves; v) búsqueda de la evidencia; vi) evaluación crítica de dicha evidencia; y vii) elaboración/redacción de las recomendaciones específicas $^{16-18}$.
Si bien durante la última década se han registrado importantes avances en los métodos utilizados para llevar a cabo cada una de dichas etapas, es en relación a las dos últimas donde se ha producido un mayor avance desde el punto de vista metodológico. Dado que los diferentes grupos que elaboran GPC juzgan de manera muy diversa la calidad de la evidencia y la fuerza de las recomendaciones, un grupo de investigadores internacionales (grupo de trabajo GRADE: 'Gradirey of Recommendations, Assessment, Development, and Evaluation') ha elaborado y refinado un método para estandarizar los procesos a través de los cuales se realizan dichos juicios: el sistema GRA$\mathrm{DE}^{19}$. Dicho método ha sido altamente influyente en la manera como actualmente se conciben los procesos de elaboración de GPC, y un gran número de organizaciones lo utiliza en sus propios procesos de elaboración. Un mayor detalle acerca del sistema GRADE será presentado en futuros artículos en esta Sección de la Revista.

La elaboración de GPC requiere el uso de una metodología rigurosa que intente minimizar el sesgo que pudiese ser introducido en cada una de sus etapas. Muchos países de altos ingresos han establecido programas formales para la elaboración de GPC, como una manera de afrontar la complejidad de estos procesos y la elevada cantidad de recursos especializados que consumen ${ }^{20}$. La mayoría de estos programas han surgido con el objetivo de mejorar la calidad y la efectividad del cuidado de la atención de salud en sus respectivos países y coinciden en la utilización de los principios de la MBE y las etapas anteriormente mencionadas para la elaboración de sus GPC. Sus diferencias tienen que ver más bien con el énfasis que ponen en los procesos de diseminación e implementación. Es así como dentro de las organizaciones más reco-

Preguntas clínicas específicas

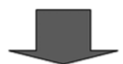

Búsqueda y análisis de la evidencia

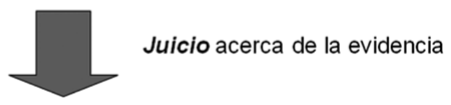

Recomendaciones para la práctica clínica

Figura 1. Etapas en la elaboración de una guía de práctica clínica. 
nocidas en el área se encuentran el New Zealand Guidelines Group (NZGG) en Nueva Zelanda, la US Preventive Service Task Force (USPSTF) en los Estados Unidos de Norteamérica, la Scottish Intercollegiate Guidelines Network (SIGN) en Escocia, el National Institute for Clinical Excellence (NICE) en Inglaterra, y el National Health and Medical Research Council (NHMRC) en Australia.

Sin embargo, contar con una infraestructura (recursos humanos y organizacionales) no garantiza que las GPC sean elaboradas bajo estándares de calidad adecuados. Con el fin de evaluar si los grupos elaboradores de GPC han utilizado métodos rigurosos que minimicen la posibilidad de sesgo, un grupo de investigadores en el área -el Grupo Colaborativo AGREE (Appraisal of Guidelines, Research, and Evaluation in Europe)- ha desarrollado un método genérico para evaluar la calidad metodológica de las $\mathrm{GPC}^{21,22}$. El instrumento AGREE evalúa tanto la calidad del reporte como la de algunos aspectos de las recomendaciones. Se trata de un instrumento que puede ser aplicado a guías de cualquier condición de salud, en sus aspectos preventivos, diagnósticos o terapéuticos. Consta de 23 preguntas, con respuestas en una escala ordinal de 1 a 7 , organizadas en seis dominios. Cada dominio intenta evaluar una dimensión separada de la calidad de las guías:

- El alcance y objetivo: el objetivo general de la guía, las preguntas clínicas específicas y la población de pacientes objetivo.

- La participación de los grupos de interés: el grado en que la guía representa los puntos de vista de los potenciales usuarios.

- El rigor en la elaboración: el proceso utilizado para recopilar y sintetizar la evidencia, los métodos para formular las recomendaciones, y la estrategia para actualizar la guía.

- La claridad y presentación: se refiere al lenguaje y formato de la guía.

- La aplicabilidad: las implicancias organizacionales, conductuales y financieras de la aplicación de la guía.

- La independencia editorial: la formulación de las recomendaciones no está sesgada por la existencia de conflictos de interés.

Este instrumento es la herramienta más ampliamente usada para la evaluación de las GPC y ha sido utilizada en por lo menos 42 estudios de 626 GPC durante los últimos 20 años $^{23}$. Recientemente se ha publicado una nueva versión del instrumento -el AGREE II- que mejora algunos problemas identificados previamente y que está comenzando a ser utilizada por los investigadores en el área ${ }^{24}$. Gran parte del material desarrollado se encuentra disponible en el sitio web de la Colaboración AGREE (http://www.agreetrust.org/), incluyendo la traducción del instrumento al español.

En Chile, un estudio publicado el año 2007, que utilizó el instrumento AGREE en GPC elaboradas para la atención primaria entre 1999 y 2004, encontró 33 documentos, 15 obtenidos tras una búsqueda manual y 18 tras una búsqueda electrónica. Sólo 9 de ellos (28\%) cumplieron con la definición de GPC hecha por el estudio y fueron incluidos en el análisis final ${ }^{25}$. Según la evaluación global cualitativa del instrumento AGREE, $66 \%$ de las GPC fueron clasificadas como no recomendadas dada su baja calidad metodológica, $23 \%$ recomendadas con modificaciones y sólo una $(11 \%)$ resultó claramente recomendada. Un estudio más reciente, evaluó con una metodología similar las GPC elaboradas entre 2005 y 2009 en el contexto de la reforma de salud chilena. Se evaluaron 60 GPC, y si bien la calidad de las guías chilenas está lejos de ser ideal, existen dimensiones relativamente bien evaluadas como "Alcance y objetivo" (82,2\% del máximo puntaje posible) y "Claridad y presentación" (68,8\%). Sin embargo, aspectos relacionados con la "Aplicabilidad" (23,3\%), y la "Participación de los grupos de interés relevantes" (37,5\%), debieran ser el foco de estrategias de mejora en el Programa de GPC de nuestro sistema de salud ${ }^{26}$.

Si bien el instrumento AGREE ha sido utilizado extensamente en el ámbito de la investigación, presenta limitaciones para su uso en la práctica clínica habitual (necesidad de, al menos, 2 evaluadores; requerimiento de tiempo dada su extensión) ya sea por profesionales de la salud u organizaciones individuales que desean evaluar cuanto "confiar" en una GPC en particular. Debido a ello es que, durante los últimos años, se han elaborado listados de estándares de más fácil uso que permitan a aquellos que aplican las GPC realizar juicios respecto a la "confiabilidad" que pueden poner en las recomendaciones publicadas en diferentes $\mathrm{GPC}^{13,27}$. Los estándares publicados por el Instituto de Medicina de los Estados Unidos de Norteamérica en 2011 han sido especialmente influyentes y fueron descritos y utilizados recientemente, en el contexto nacional, para la evaluación de la guía 
Elaboración e implementación de guías clínicas - T. Pantoja et al

para el Diagnóstico y Manejo Clínico de Casos de Influenza elaborada por el Ministerio de Salud ${ }^{28}$.

Independientemente de cuál sea el método utilizado para evaluar el producto final del proceso de elaboración de GPC, es importante que dicha información sea utilizada para mejorar progresivamente dichos procesos y obtener GPC "más confiables" que potencialmente puedan mejorar los procesos de toma de decisiones clínicas en nuestro sistema de salud.

\section{Difusión, diseminación e implementación de GPC}

Sin embargo, no basta con disponer de GPC de alta calidad metodológica (o "más confiables"), sino que ellas debieran ser implementadas a través de estrategias efectivas para obtener cambios en la práctica clínica y mejoramiento en los resultados de salud de los pacientes ${ }^{29,30}$. La evidencia respecto a los efectos de las diferentes estrategias de implementación muestra resultados modestos y altamente variables ${ }^{31,32}$. En general, no existen "recetas mágicas" que sean aplicables a todas las situaciones. Si bien algunas intervenciones son más efectivas que otras, se deben considerar múltiples elementos antes de decidir la estrategia de implementación más adecuada para un determinado contexto $^{33}$. Es importante destacar, además, que numerosas publicaciones han resaltado el rol de las barreras y facilitadores tanto a nivel individual como organizacional para la implementación de las $\mathrm{GPC}^{34-38}$.

La Tabla 1 muestra un resumen de la efectividad de algunas intervenciones para implementar GPCs, así como su definición operacional. Cabe mencionar que para las sesiones educativas los efectos de mayor tamaño se relacionaron con las tasas de asistencia a las sesiones y el grado de interactividad de ellas ${ }^{39}$. En el caso de la auditoría y retroalimentación, los mayores efectos se observaron en aquellos casos con adherencia basal más baja ${ }^{40}$. Finalmente, en el caso de los recordatorios la mayoría de los estudios han evaluado intervenciones relativamente simples dirigidas a modificar comportamientos de baja complejidad (por ejemplo, prescripción) ${ }^{41}$.

En Chile, pese a la importante cantidad de GPC elaboradas, principalmente en el contexto de la reforma de salud, existen pocas publicaciones que aborden los temas del diseño y evaluación de estrategias de implementación. Se han evaluado los cumplimientos de recomendaciones incluidas en GPC del Régimen de Garantías Explícitas, pero no en el contexto de estrategias específicas de implementación diseñadas para contextos clínicos particulares ${ }^{42,43}$. La poca información existente respecto a este ámbito proviene de la atención primaria. Soto y cols evaluaron los efectos de una estrategia participativa basada en el uso de líderes de opinión y métodos de consenso en la implementación de una guía clínica de control prenatal, encontrando mejoría en la adherencia a prácticas

Tabla 1. Definición y efectividad de algunas estrategias de implementación de GPC

\section{Estrategia}

Distribución de materiales educativos: distribución de recomendaciones impresas o publicadas para el cuidado clínico, sea en la forma de guías, material audio-visual, o publicaciones electrónicas

Sesiones educativas: participación en conferencias, clases, seminarios o talleres

Líderes de opinión local: uso de profesionales sanitarios nominados con "influyentes educacionalmente" por sus pares

Auditoría \& retroalimentación: cualquier resumen de desempeño clínico en un período específico de tiempo

Recordatorios: información relacionada con un paciente que es entregada verbalmente, en forma escrita o a través de una pantalla de computador y que está diseñada para que el profesional sanitario recuerde algún aspecto específico del cuidado del paciente

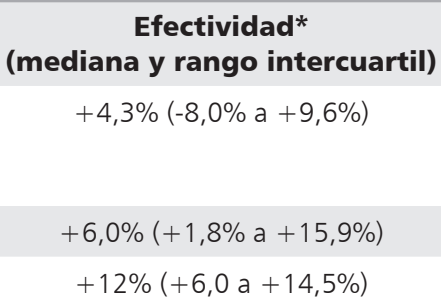

Efectividad* (mediana y rango intercuartil)

$+4,3 \%(-8,0 \%$ a $+9,6 \%)$

$+6,0 \%(+1,8 \% a+15,9 \%)$

$+12 \%(+6,0 a+14,5 \%)$

$+5,0 \%(+3,0 \% a+11 \%)$

$+14,1 \%(-1,0 \% a+34 \%)$

*valores correspondientes a desenlaces dicotómicos de proceso: mejoramiento en la adherencia a recomendaciones específicas. 
basadas en evidencia ${ }^{44}$. Asimismo, García y cols, utilizando un diseño controlado antes-después, evaluaron los efectos de una intervención múltiple (materiales impresos, recordatorios, reuniones de consenso) en la implementación de una guía clínica de Diabetes Mellitus, encontrando mejoría en una serie de indicadores de calidad ${ }^{45}$.

Sin embargo, el área de la diseminación/implementación de las GPC no ha recibido una atención de similar magnitud a los esfuerzos puestos en la elaboración de ellas. En muchos casos, se ha asumido que las organizaciones de salud en forma individual deben hacerse cargo de dichos esfuerzos y no se han ofrecido orientaciones específicas para iniciar el proceso.

\section{Discusión}

Las GPC son herramientas que intentan promover el uso de la evidencia científica en las decisiones clínicas, con una especial consideración del contexto en donde dichas decisiones son realizadas. Por ello, su elaboración e implementación son procesos que están fuertemente interconectados tanto desde el punto de vista conceptual como práctico y que requieren la inversión de una cantidad importante de recursos. Asimismo, dichos procesos constituyen desafíos metodológicos de gran complejidad debido a que se deben abordar contenidos de diferentes disciplinas y áreas, como son los resúmenes de evidencia y revisiones sistemáticas, el uso de métodos de consenso para la interpretación de dicha evidencia, y las teorías de cambio a nivel individual y organizacional para su implementación. Aunque nuestro sistema sanitario ha realizado importantes esfuerzos para la elaboración y potencial diseminación de GPC, todavía existen una serie de aspectos potencialmente mejorables en dichos procesos.

Actualmente, un área de especial interés que permitiría potencialmente vincular los procesos de elaboración e implementación es aquel relacionado con los atributos de las recomendaciones de las GPC que hacen más probable su implementación en la práctica. Si bien dichas características han sido estudiadas desde hace un tiempo, sólo recientemente han comenzado a ser evaluadas más consistentemente, con la finalidad de modificar los procesos utilizados en su elaboración para hacer sus recomendaciones más "implementables" $46-48$.

Otra área de interés es la relacionada con la inclusión de procesos relativamente estandarizados de valoración de la calidad de la evidencia y fuerza de las recomendaciones (por ejemplo, el sistema GRADE) en la elaboración de GPC. En teoría, esto permitiría un uso más sistemático y transparente de la evidencia existente para la formulación de las recomendaciones, y contribuiría a una mejor adherencia a dichas recomendaciones por parte de los profesionales sanitarios.

Finalmente, uno de los aspectos al que se ha dado relativamente poca consideración es aquel relacionado con la existencia de conflictos de intereses entre los integrantes de los grupos elaboradores de las GPC. Si bien esto no ha sido evaluado en detalle en el contexto nacional, la literatura internacional muestra que su prevalencia en los grupos elaboradores y el sub-reporte por parte de sus integrantes es alto ${ }^{49,50}$. Por lo tanto, este debiera ser otro aspecto a considerar con el fin de aumentar la "confiabilidad" de las recomendaciones incluidas en las GPC elaboradas en nuestro país.

Resumiendo, se han presentado aspectos generales de los procesos de elaboración e implementación de GPC, junto con parte de la literatura existente a nivel nacional. Un mejor conocimiento y discusión de dichos procesos nos permitirá -potencialmente- avanzar en esta área, promoviendo decisiones sanitarias mejor informadas por la evidencia científica y mejores resultados de salud en nuestros pacientes.

\section{Referencias}

1. Committe on Quality of Health Care in America IoM. Crossing the quality chasm: a new health system for the 21st century. Washington: National Academy Press; 2001.

2. Lord Darzi. High Quality Care for All: NHS Next Stage Review Final Report. Norwich: The Stationery Office; 2008.

3. Orszag PR, Emanuel EJ. Health care reform and cost control. N Engl J Med 2010; 363 (7): 601-3.

4. Evidence-Based Medicine Working Group. Evidencebased Medicine: a new approach to teaching the practice of medicine. JAMA 1992; 268: 2420-5.

5. Sackett DL, Straus SE, Richardson WS, Rosenberg WMC, Haynes RB. Evidence-Based Medicine. How to Practice 
and Teach EBM. 2nd ed. Edinburgh: Churchill Livingstone; 2000.

6. Guyatt GH, Rennie D. Users' Guides to the Medical Literature. A Manual for Evidence-Based Clinical Practice. Chicago: AMA Press; 2002.

7. McColl A, Smith H, White P, Field J. General practitioners' perceptions of the route to evidence based medicine: a questionnaire survey. BMJ 1998; 316: 361-5.

8. Al OM, Khader Y, Jadallah K, Dauod AS, Al-Shdifat AA, Khasawneh NM. Evidence-based medicine among hospital doctors in Jordan: awareness, attitude and practice. J Eval Clin Pract 2009; 15 (6): 1137-41.

9. van DN, Hooft L, Wieringa-de WM. What are the barriers to residents' practicing evidence-based medicine? A systematic review. Acad Med 2010; 85 (7): 1163-70.

10. Young JM, Ward JE. Evidence-based medicine in general practice: beliefs and barriers among Australian GPs. J Eval Clin Pract 2001; 7: 201-10.

11. Pantoja T. Medicina General Familiar y Medicina Basada en Evidencias: ¿una combinación posible? Rev Chil Medicina Familiar 2000; I (4): 5-7.

12. Institute of Medicine (Field MJ \& Lohr KN eds). Guidelines for Clinical Practice. From Development to Use. Washington DC: National Academy Press; 1992.

13. Institute of Medicine. Clinical Practice Guidelines We Can Trust. Washington DC: The National Academies Press; 2011.

14. Thorsen T, Mäkelä M. Changing Professional Practice. Theory and Practice of Clinical Guidelines Implementation. Copenhagen: Danish Institute for Health Services Research and Development; 1999.

15. Greenhalgh T, Robert G, Macfarlane F, Bate P, Kyriakidou O. Diffusion of innovations in service organizations: systematic review and recommendations. Milbank Q 2004; 82 (4): 581-629.

16. New Zealand Guidelines Group. Handbook for the preparation of explicit evidence-based clinical practice guidelines. Wellington: New Zealand Guidelines Group; 2001.

17. Scottish Intercollegiate Guidelines Network. Sign 50: A guideline developer's handbook. Edinburgh: Scottish Intercollegiate Guidelines Network; 2008.

18. National Institute for Health and Clinical Excellence. The guidelines manual. London: National Institute for Health and Clinical Excellence; 2009.

19. Guyatt GH, Oxman AD, Vist GE, Kunz R, Falck-Ytter Y, Alonso-Coello P, et al. GRADE: an emerging consensus on rating quality of evidence and strength of recommendations. BMJ 2008; 336 (7650): 924-6.

20. Burgers JS, Grol R, Klazinga NS, Mäkelä M, Zaat J, for the AGREE Collaboration. Towards evidence-based clinical practice: an international survey of 18 clinical guideline programs. Int J Qual Health Care 2003; 15: 31-45.

21. The Appraisal of Guidelines Research and Evaluation in Europe (AGREE) Collaborative Group. Development and validation of an international appraisal instrument for assessing the quality of clinical practice guidelines: the AGREE project. Qual Saf Health Care 2003; 12: 1823.

22. Vlayen J, Aertgeerts B, Hannes K, Sermeus W, Ramaekers D. A systematic review of appraisal tools for clinical practice guidelines: multiple similarities and one common deficit. Int J Qual Health Care 2005; 17 (3): 235-42.

23. Alonso-Coello P, Irfan A, Sola I, Gich I, Delgado-Noguera $\mathrm{M}$, Rigau $\mathrm{D}$, et al. The quality of clinical practice guidelines over the last two decades: a systematic review of guideline appraisal studies. Qual Saf Health Care 2010; 19 (6): 1-7.

24. Brouwers MC, Kho ME, Browman GP, Burgers JS, Cluzeau F, Feder G, et al. AGREE II: advancing guideline development, reporting and evaluation in health care. J Clin Epidemiol 2010; 63 (12): 1308-11.

25. Pantoja T, Strain H, Valenzuela L. Guías de práctica clínica en atención primaria: Una evaluación crítica. Rev Med Chile 2007; 135 (10): 1282-90.

26. Pantoja T, Valenzuela L, Léniz J, Castañón C. Guías de Práctica Clínica en el Régimen de Garantías en Salud: una evaluación crítica de su calidad. Rev Med Chile 2012; 140: 1391-400.

27. Qaseem A, Forland F, Macbeth F, Ollenschläger G, Phillips S, for the Board of Trustees of the Guidelines International Network. Guidelines International Network: Toward International Standards for Clinical Practice Guidelines. Ann Intern Med 2012; 156: 525-31.

28. Neumann I, Cifuentes L. Hacia guías de práctica clínica más confiables. La Guía para el manejo de la Influenza del Ministerio de Salud. Rev Med Chile 2012; 140: 16367.

29. Grol R. Successes and failures in the implementation of evidence-based guidelines for clinical practice. Medical Care 2001; 39 (8 (Suppl 2)): II.

30. Grol R, Grimshaw JM. From best evidence to best practice: effective implementation of change in patients' care. Lancet 2003; 362: 1225-30.

31. Grimshaw J, Eccles M, Thomas R, MacLennan G, Ramsay C, Fraser C, et al. Toward evidence-based quality improvement. Evidence (and its limitations) of the effectiveness of guideline dissemination and implementation strategies 1966-1998. J Gen Intern Med 2006; 21 (Suppl 2): S14-S20.

32. Grimshaw JM, Eccles MP, Lavis JN, Hill SJ, Squires JE. 
Elaboración e implementación de guías clínicas - T. Pantoja et al

Knowledge translation of research findings. Implement Sci 2012; 7: 50.

33. Oxman AD, Thomson MA, Davis DA, Haynes RB. No magic bullets: a systematic review of 102 trials of interventions to improve professional practice. CMAJ 1995; 153 (10): 1423-31.

34. Burgers JS, Grol RPTM, Zaat J, Spies TH, Van der Bij AK, Mokkink H. Characteristics of effective clinical guidelines for general practice. Br J Gen Pract 2003; 53: 15-9.

35. Flottorp S, Oxman AD. Identifying barriers and tailoring interventions to improve the management of urinary tract infections and sore throat: A pragmatic study using qualitative methods. BMC Health Services Research 2003; 3: 1-10

36. Bosch M, van der WT, Wensing M, Grol R. Tailoring quality improvement interventions to identified barriers: a multiple case analysis. J Eval Clin Pract 2007; 13 (2): 161-8.

37. Carlsen B, Glenton C, Pope C. Thou shalt versus thou shalt not: a meta-synthesis of GPs' attitudes to clinical practice guidelines. Br J Gen Pract 2007; 57: 971-8.

38. Lugtenberg M, Burgers JS, Besters CF, Han D, Westert GP. Perceived barriers to guideline adherence: A survey among general practitioners. BMC Fam Pract 2011; 12: 98.

39. Forsetlund L, Bjorndal A, Rashidian A, Jamtvedt G, O'Brien MA, Wolf F, et al. Continuing education meetings and workshops: effects on professional practice and health care outcomes (Review). Cochrane Database Syst Rev 2009; 2: CD003030.

40. Jamtvedt G, Young JM, Kristoffersen DT, Thomson O'Brien MA, Oxman AD. Audit and feedback: effects on professional practice and health care outcomes (Review). Cochrane Database Syst Rev 2006; 2: CD000259.

41. Shojania KG, Jennings A, Mayhew A, Ramsay CR, Eccles MP, Grimshaw J. The effects of on-screen, point of care computer reminders on processes and outcomes of care. Cochrane Database Syst Rev 2009; 3: CD001096.

42. Sánchez H, Albala C, Dangour AD, Uauy R. Cumpli- miento de la guía clínica de manejo ambulatorio de la neumonía adquirida en la comunidad en personas mayores en centros de atención primaria de salud de Santiago. Rev Med Chile 2009; 137 (12): 1575-82.

43. Wang Y, Álvarez G, Salinas R, Ramírez G, Catalán M, Díaz C. Análisis del cumplimiento de la Guía Clínica del AUGE en pacientes con accidente cerebrovascular isquémico: la utilización de tecnología sanitaria de diagnóstico para prevención secundaria en un hospital público. Rev Med Chile 2011; 139 (6): 697-703.

44. Soto M, Valenzuela L, Pantoja T. Implementación de una guía de práctica clínica en control prenatal en el Cesfam El Roble, La Pintana. Rev Chil Medicina Familiar 2003; 4 (3): 98-102.

45. García L, Pantoja T, Undurraga JP. Mejorando la calidad del cuidado sanitario en Diabetes Mellitus 2: implementación de una guía de práctica clínica en atención primaria. Rev Chil Medicina Familiar 2006; 7 (1): 31-8.

46. Grol R, Dalhuijsen J, Thomas S, Veld Ci, Rutten G, Mokkink $\mathrm{H}$. Attributes of clinical guidelines that influence use of guidelines in general practice: observational study. BMJ 1998; 317: 858-61.

47. Kastner M, Estey E, Bhattacharyya O. Better guidelines for better care: enhancing the implementability of clinical practice guidelines. Expert Rev Pharmacoecon Outcomes Res 2011; 11 (3): 315-24.

48. Gagliardi AR, Brouwers MC, Palda VA, Lemieux-Charles L, Grimshaw JM. How can we improve guideline use? A conceptual framework of implementability. Implement Sci 2011; 6: 26.

49. Neuman J, Korenstein D, Ross JS, Keyhani S. Prevalence of financial conflicts of interest among panel members producing clinical practice guidelines in Canada and United States: cross sectional study. BMJ 2011; 343: d5621.

50. Norris SL, Holmer HK, Ogden LA, Burda BU. Conflict of Interest in Clinical Practice Guideline Development: A Systematic Review. PLoS One 2011; 6 (10): e25153. 